\title{
Effects of functional electrical stimulation on the joints of adolescents with spinal cord injury
}

\author{
R Betz ${ }^{1}$, B Boden ${ }^{2}, \mathrm{R}$ Triolo ${ }^{3}, \mathrm{M}$ Mesgarzadeh ${ }^{2}, \mathrm{E} \mathrm{Gardner}^{4}$ and R Fife ${ }^{5}$ \\ ${ }^{1}$ Shriners Hospital, 8400 Roosevelt Boulevard, Philadephia, PA 19152, USA; ${ }^{2}$ Temple University Hospital, 3401 \\ North Broad Street, Philadelphia, PA 19140, USA; ${ }^{3}$ Veterans Administration Medical Center, 10701 East Boulevard, \\ Cleveland, OH 44106, USA; ${ }^{4}$ Johns Hopkins University, Dept. of Otolaryngology, 601 N. Caroline Street, Baltimore, \\ MD 21287 USA; ${ }^{5}$ Fesler Hall 302, 1120 South Drive, Indianapolis, IN 46202-5114, USA
}

\begin{abstract}
Nineteen adolescent subjects with complete spinal cord injuries resulting in paraplegia or tetraplegia participated in a functional electrical stimulation (FES) program consisting of computerized, controlled exercise and/or weight bearing. The effects of stimulated exercise and standing/ walking on the lower extremity joints were prospectively studied. Plain radiographs and MRIs were obtained prior to and following completion of the exercise and standing and walking stages. In addition, the joints of five subjects were studied with synovial biopsies, arthroscopy, and the analysis of serum and synovial fluid for a 550000 dalton cartilage matrix glycoprotein (CMGP). Pre-exercise joint abnormalities secondary to the spinal cord injury improved following the stimulation program. None of the subjects developed Charcot joint changes. Upon standing with FES, one subject with poor hip coverage prior to participation developed hip subluxation which required surgical repair. No other detrimental clinical effects occured in the lower extremity joints of subjects participating in an FES program over a 1year period.
\end{abstract}

Keywords: functional electrical stimulation; spinal cord injury; paraplegia; tetraplegia; exercise; standing

\section{Introduction}

Joint immobilization in humans and animals is associated with articular cartilage changes including altered proteoglycan synthesis, fibrofatty proliferation within the joint, and thinning of the cartilage..$^{1,2,3}$ Reversal of articular cartilage abnormalities can occur and has been documented histologically in animal studies using continuous passive motion. ${ }^{4}$ Joint motion in the absence of normal loading, however, has been shown to incompletely reverse the changes in proteoglycan synthesis. ${ }^{5}$ In canine articular cartilage, complete reversal of atrophic changes has been documented after remobilization with weight bearing. ${ }^{6,7}$

Only one case was identified in the literature demonstrating specific changes in a human joint after traumatic spinal cord injury. ${ }^{1}$ Histological examination revealed osteoporotic bone, normal articular cartilage, and normal subchondral plates in a lower extremity joint specimen of a patient with SCI after immobilization due to paralysis. Fibrofatty connective tissue was present in the space between the articulating surfaces of the patella and the femoral condyles. This fibrous connective tissue appeared to blend into the articular cartilage.

A major concern in the remobilization of patients

Correspondence: $\mathrm{R}$ Betz with spinal cord injury resulting in both sensory and motor deficits is the potential for developing neuropathic or Charcot joints. Neuropathic joint changes have been noted in patients with diabetes, tabes dorsalis, syringomyelia, and congenital insensitivity to pain. ${ }^{8}$ A review of the literature revealed no extraaxial neuropathic changes in patients with spinal cord injury, which is most likely due to the limited physical activity of these patients. However, with remobilization and weight bearing in the form of exercise, standing and walking with FES, the potential for harmful effects to the joints exists.

The purpose of this study was to assess the preexisting changes in the lower extremity joints of subjects with spinal cord injuries, and to prospectively collect data on the effects of exercise, standing and walking with FES on the paralyzed and insensate joints of the lower extremities.

\section{Materials and methods}

Researchers at the Philadelphia Unit of Shriners Hospitals are investigating the use of FES in the lower extremities of subjects with spinal cord injury to provide standing and ambulatory skills. One facet of the research involves the study of FES and its effects on the joints. 


\section{Implementation of FES}

Our technique of FES involves delivery of electrical stimulation to nerves via percutaneously implanted intramuscular electrodes.9,10 For lower extremity applications, the electrodes are inserted under sterile conditions in the operating room without anesthesia because of absent sensation. Electrodes are implanted near the quadriceps motor point (femoral nerve) for subjects in the exercise (conditioning) program. An additional 10 to 15 electrodes are inserted in the lower extremity muscles of subjects progressing to the standing program and up to 48 electrodes for those advancing to the walking program. ${ }^{9}$ A research-grade 48-channel stimulator was utilized. The electrical stimulation parameters included a biphasic asymmetrical waveform at a frequency of $20 \mathrm{~Hz}$, amplitude of $20 \mathrm{~mA}$, with a pulse duration of $50-150 \mu \mathrm{sec}$.

\section{Subjects}

Between 1986 and 1992, 19 subjects participated in the FES lower extremity program. Their average age at the beginning of the study was 17 years (range 13 to 19 years). There were 12 male and 7 female subjects. All of the subjects had sustained a complete traumatic spinal cord injury. The level of injury was either cervical or thoracic for those in the exercise program and thoracic for those participating in the standing and walking program. The average time from the injury to the initiation of the FES program was 38 months (range 11 to 117 months).

\section{Exercise}

The 8-week exercise program was designed to build the strength and endurance of the quadriceps musculature. The subjects exercised for endurance for either 1 or 2 hours per day 5 times per week, depending on which conditioning protocol they were following. Strength training included incremental resistance with weights starting at $\times 2 \mathrm{lbs}$. Progressive resistance required three sets of 10 knee extensions with weights that could be lifted without loss of range of motion. The maximum weight lifted in one case was equal to half the subject's body weight. The exercise program was designed to promote reversal of muscle atrophy prior to the onset of weight bearing.

\section{Standing and walking}

Following the exercise program, the subjects who advanced to the standing program had additional electrodes implanted to stimulate the gluteus maximus, gluteus medius, the paraspinal muscles, and the individual heads of the quadriceps muscles. The subjects who progressed to the walking stage had additional electrodes implanted into the hip flexors (iliopsoas, tensor fascia lata, sartorius, gracilis) and hamstring muscles. Frequency and duration of standing and walking varied from subject to subject. Continuous standing times could be as short as two minutes or as long as 20 min per trial, depending on the strength and endurance of the subject. Standing trials separated by rest breaks were repeated several times during each standing session, which occurred 4 to 5 times per week during the 3-month standing program. Subjects advancing to the walking phase underwent standing and stepping with FES for an additional 6 months. Walking sessions occurred 3 to 4 times per week and generally consisted of 15 to $30 \mathrm{~min}$ of intense activity. By the end of the program, subjects were routinely able to complete 5 to 10 repetitions of approximately 20 meters at speeds approaching $25 \%$ of normal. Further details of the FES exercise, standing and walking program can be found elsewhere. ${ }^{11}$

\section{Studies}

All 19 subjects participated in the exercise (conditioning) program. In addition to close clinical observation, the subjects underwent pre-and post-exercise plain radiographs and MRIs of their knees. The plain films (read by a radiologist [MM] and independently reviewed by one of the authors $[\mathrm{BB}]$ ) were examined for evidence of destructive changes such as resorptive osteolysis, subchondral lucencies, juxtaarticular fractures, osteophytes, joint space narrowing, or Charcot joint findings. ${ }^{12}$ It has been reported that MRI studies can reveal destructive changes earlier than radiography. ${ }^{13}$ Therefore, pre- and post-exercise MRIs were reviewed by an experienced radiologist (MM) for effusions, bone contusions, articular cartilage changes, meniscal pathology, and/or ligamentous changes.

Of the 19 subjects in the exercise program, 11 progressed to the standing program and eight of the 11 to the walking stage. Seven of the exercise subjects had tetraplegia and were thus not considered for the walking phase. At the conclusion of the exercise program, plain erect radiographs of the hips, knees, and ankles, as well as MRIs of the knees and ankles, were obtained. For those who completed the standing and/or walking programs, these studies were repeated and examined for any changes in the joints.

MRI is not as sensitive as arthroscopy for detecting Grade I and Grade II articular cartilage degeneration. ${ }^{14}$ In this study, we performed arthroscopy of knees and ankles bilaterally in the first five subjects pre-exercise, post-exercise, and at the conclusion of the standing and/or walking program. At the time of the knee arthroscopies, synovial biopsies were obtained and sent for pathological analysis. Due to the lack of abnormal findings and the potential for iatrogenic articular cartilage damage, the arthroscopies were discontinued after the first five subjects.

The articular cartilage was also assessed for biochemical changes by measuring cartilage matrix glycoprotein (CMGP) in the synovial fluid of those who underwent arthroscopy and in the serum of subjects who would consent to a venous sample. CMGP is a noncollagenous, nonproteoglycan cartilage protein synthesized by hyaline cartilage and fibrocartilage. ${ }^{15,16}$ CMGP has a short half-life $(48-72 \mathrm{~h})$ in cartilage. ${ }^{17}$ It has been noted in the serum and 
synovial fluid of canines with experimentally induced osteoarthritis ${ }^{18}$ and in humans with cartilage degeneration. ${ }^{19,20} \mathrm{CMGP}$ is not present in normal canine or human knees. ${ }^{18}$ CMGP was measured by enzymelinked immunofluorescent assay (ELISA) using a monoclonal antibody (AR S 5 G-3) to CMGP. ${ }^{18}$ Serum samples were obtained serially from six subjects and synovial fluid from four subjects.

A summary of the subjects' data and interval measurements is presented in Table 1 .

\section{Results}

Pre-existing joint status and subsequent effect of FES on the joints

Prior to the onset of the exercise program, one subject (Case 5) had right knee ligament instability (both cruciates and the lateral collateral ligament) on clinical examination which did not cause any problems throughout the study period. The radiographic studies revealed diffuse osteopenia in all subjects. Demineralization (seen on plain radiographs) was most evident in the metaphyses of the long bones and did not change over the course of the study. One subject (Case 16) had radiographic signs of a hip at risk for subluxation. The hip dislocated upon standing, and this will be discussed in detail later. Slight narrowing of the knee joint space was seen in one subject (Case 10) on pre-exercise radiographs as compared to normal subjects with bilateral standing knee radiographs. This remained unchanged in subsequent studies taken post- exercise and post standing and walking. Knee radiographs from one subject (Case 5) showed posttraumatic changes consistent with her clinical ligament instability, and these findings did not change in followup studies postexercise and post standing and walking.

\section{Pre-existing knee abnormalities revealed by MRI}

Pre-exercise MRIs of the knee revealed a small, clinically nonpalpable effusion in 14 of 32 knees (Figure 1A and B). Follow-up MRI obtained postexercise showed no change in 10 knees and resolution of the effusion in 4 . No effusion was palpable at the time of MRI. One subject (Case 5) had tears of both cruciates, of the lateral collateral ligaments, and of both menisci (right knee) on the MRI, which confirmed the clinical examinations noted above. In another subject (Case 12), a clinically undetected partial tear of the anterior cruciate ligament, as well as a tear of the lateral meniscus, were revealed and no deterioration was seen on subsequent MRIs or on clinical examination. Thinning of the articular cartilage of the left medial femoral condyle was present in one subject (Case 11 ) on MRI pre-exercise and returned to normal on the post-exercise MRI. Analysis of ankle MRIs revealed 4 subjects with minimal ankle effusions, all of which resolved by the time of the post-exercise studies. None of the effusions detected on MRI was clinically palpable.

Arthroscopic examination of the knees pre-exercise and post-exercise revealed no abnormalities. Arthroscopic examination of the ankles pre-exercise revealed two subjects (Case 11 bilaterally and Case 15 right) in

Table 1 Measurement intervals*

\begin{tabular}{|c|c|c|c|c|c|c|c|}
\hline Case no. & Initials & Age $(y r s)^{* *}$ & $\begin{array}{l}\text { Level of } \\
\text { injury }\end{array}$ & $\begin{array}{l}\text { Months** } \\
\text { post injury }\end{array}$ & $\begin{array}{c}\text { Pre-exercise } \\
\text { (baseline) }\end{array}$ & $\begin{array}{c}\text { Post-exercise } \\
(8-12 \text { weeks })\end{array}$ & $\begin{array}{c}\text { Post stand/walk } \\
\text { (approx. } 12 \text { mos.) }\end{array}$ \\
\hline 1 & TB & 18 & $\mathrm{C} 5$ & 25 & $X$ & $X$ & \\
\hline 2 & $\mathrm{JB}$ & 19 & $\mathrm{C} 5$ & 34 & $\mathrm{X}$ & $X$ & \\
\hline 3 & BB & 19 & C6 & 20 & $\mathrm{X}$ & $X$ & \\
\hline 4 & $\mathrm{CC}$ & 15 & $\mathrm{C} 5$ & 11 & X & $X$ & \\
\hline 5 & $\mathrm{BK}$ & 19 & T5 & 29 & $X^{(1,4)}$ & $X^{(1,4)}$ & $X^{(1,4)}$ \\
\hline 6 & JM1 & 19 & $\mathrm{C} 7$ & 40 & X & X & \\
\hline 7 & JM2 & 18 & $\mathrm{C} 5$ & 13 & $X$ & $\mathrm{X}$ & \\
\hline 8 & $\mathrm{CS}$ & 15 & C6 & 61 & $\mathrm{X}$ & $\mathrm{X}$ & \\
\hline 9 & $\mathrm{CT}$ & 19 & $\mathrm{~T} 8$ & 13 & $X^{(1,4)}$ & $X^{(4)}$ & $X^{(4)}$ \\
\hline 10 & KB & 17 & $\mathrm{~T} 4$ & 28 & $X^{(1,4)}$ & $X^{(1,4)}$ & $X^{(1,4)}$ \\
\hline 11 & $\mathrm{~TB}$ & 13 & $\mathrm{~T} 4$ & 117 & $X^{(1,2,3,4,5)}$ & $X^{(1,2,3,4,5)}$ & $X^{(1,2,3,5)}$ \\
\hline 12 & $\mathrm{JC}$ & 16 & T5 & 29 & $X^{(1,2,3,4,5)}$ & $X^{(1,2,3,4,5)}$ & $X^{(1,2,3,4,5)}$ \\
\hline 13 & $\mathrm{LC}$ & 13 & $\mathrm{~T} 12$ & 61 & $X^{(1,4)}$ & $X^{(1,4)}$ & $X^{(1,4)}$ \\
\hline 14 & $\mathrm{RH}$ & 17 & $\mathrm{~T} 7$ & 21 & $X^{(1,2,3,4,5)}$ & $X^{(1,2,3,4,5)}$ & $X^{(1,2,3,4,5)}$ \\
\hline 15 & $\mathrm{MM}$ & 16 & T5 & 28 & $X^{(1,2,3,5)}$ & $X^{(1,2,3,4,5)}$ & $X^{(1,2,3,5)}$ \\
\hline 16 & MR & 15 & $\mathrm{~T} 8$ & 114 & $X^{(1,4)}$ & $X^{(1)}$ & $X^{(1,4)}$ \\
\hline 17 & TS & 18 & $\mathrm{~T} 11$ & 25 & $\mathrm{X}^{(1,4)}$ & $X^{(1,4)}$ & $\mathrm{X}^{(1,4)}$ \\
\hline 18 & $\mathrm{CT}$ & 13 & T6 & 15 & $X^{(1,2,3,4,5)}$ & $\mathrm{X}^{(1,2,3,4,5)}$ & $X^{(1,2,3,4,5)}$ \\
\hline 19 & $\mathrm{AH}$ & 13 & $\mathrm{~T} 1$ & 36 & X & X & X \\
\hline
\end{tabular}

${ }^{*} \mathrm{X}=$ All subjects had plain radiographs and MRIs of the knees pre-exercise, post exercise, and post-standing and/or walking. Additional tests are denoted in parentheses. ${ }^{* *}$ At beginning of participation in FES program. $1=$ MRI ankles; $2=$ Arthroscopy knees/ankles; 3 = Synovial biopsies; 4 = Serum CMGP; 5= Synovial fluid CMGP 


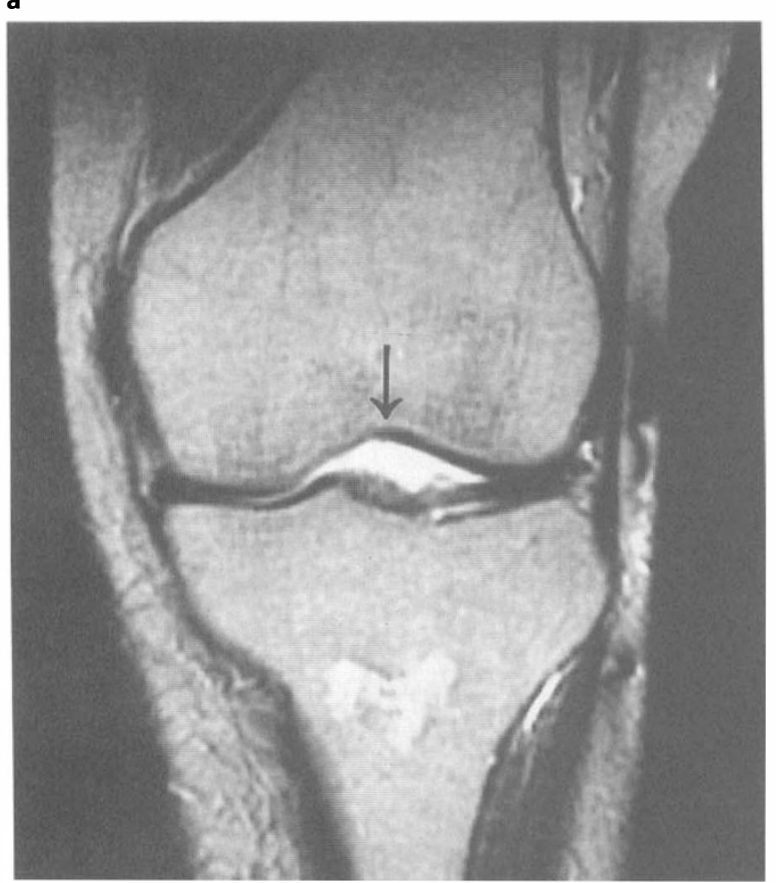

b

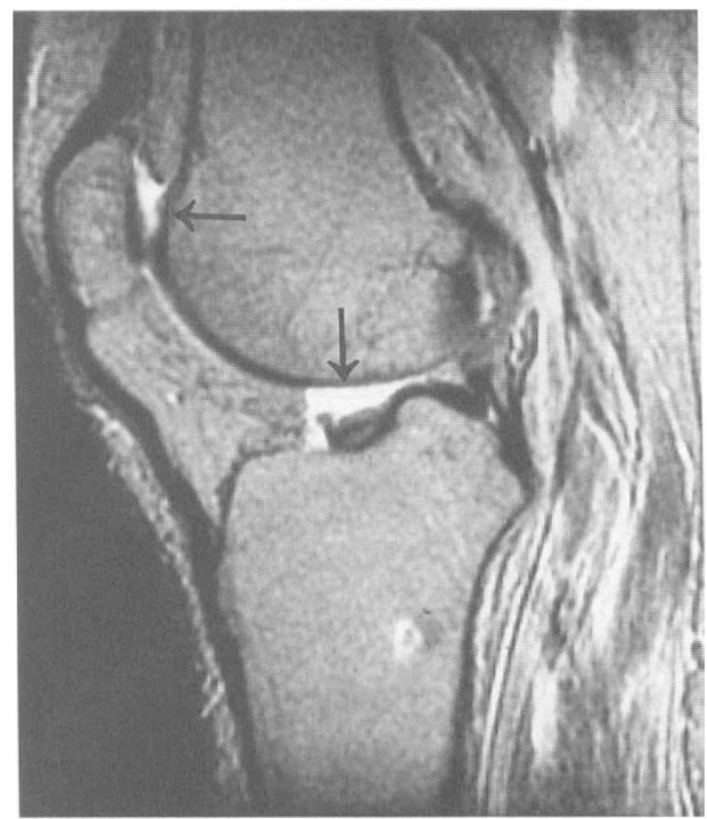

Figure 1 (a). A coronal T2 weighted image of one subject's knee (Case 10). Note the increased signal in the joint space consistent with a small effusion (arrow). Also noted in the proximal tibia is a fibrous defect. (b) This is a sagittal MRI of the same knee, again showing increased signal consistent with a small effusion in the postpatellar area and the midline of the joint. Again, a fibrous defect is noted in the proximal tibia

whom there were cartilage irregularities of the tali (chondromalacia). The cartilage surface of one subject (Case 11) was normal in the post-exercise arthroscopic exam, while the other subject's ankle (Case 15) remained unchanged.

Synovial biopsies obtained during arthroscopy of the knees prior to FES exercise revealed reactive fibrosis, neovascularization and myxomatous changes in two subjects (Cases 14 and 15, both bilaterally). In one subject (Case 15) these abnormalities were absent on the two subsequent arthroscopies post-exercise and post standing and walking. In Case 14 the abnormal findings disappeared in the right knee by the time of the post-exercise arthroscopy and disappeared in the left knee at the time of the post standing and walking arthroscopy.

Analysis of the subjects' serum for CMGP by ELISA was available for ten of the 19 subjects. Venipuncture in the arms was refused by the other nine subjects and femoral vein puncture carried too high a risk of venous thrombosis. One of the 10 patients had abnormal values of over $20 \mu \mathrm{g} \mathrm{ml}^{-1}$ pre-

Table 2 Serum CMGP in UG/ML

\begin{tabular}{|c|c|c|c|c|}
\hline Patient & Case no. & Pre-exercise & Post-exercise & Post-stand/walk \\
\hline BK & 5 & 0 & 0 & NA \\
\hline $\mathrm{CT}$ & 9 & 0 & 0 & 0 \\
\hline $\mathrm{KB}^{1}$ & 10 & 31.6 & 37.2 & 27.7 \\
\hline TB & 11 & 0 & 0 & 0 \\
\hline $\mathrm{JC}$ & 12 & 0 & 0 & 0 \\
\hline $\mathrm{LC}$ & 13 & 0 & 0 & 0 \\
\hline $\mathrm{RH}$ & 14 & 0 & 0 & 0 \\
\hline MR & 16 & 18.5 & NA & 12.6 \\
\hline $\mathrm{TS}^{2}$ & 17 & 0 & 0 & 38.7 \\
\hline CT & 18 & 0 & 23 & 0 \\
\hline
\end{tabular}

$\mathrm{NA}=$ Not available. ${ }^{1}$ No articular damage seen on radiographs or MRIs; patient has been followed for 36 months. ${ }^{2}$ This patient had a deltoid ligament sprain 
a

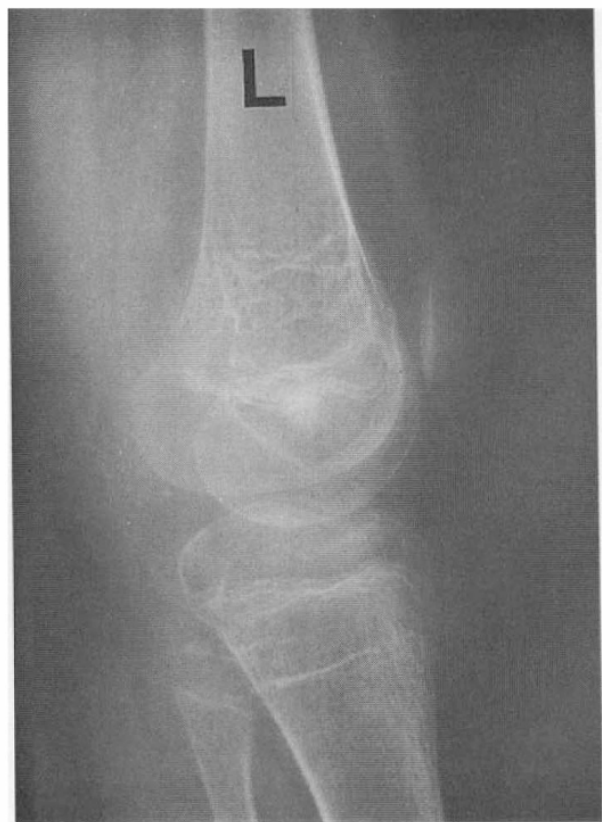

C

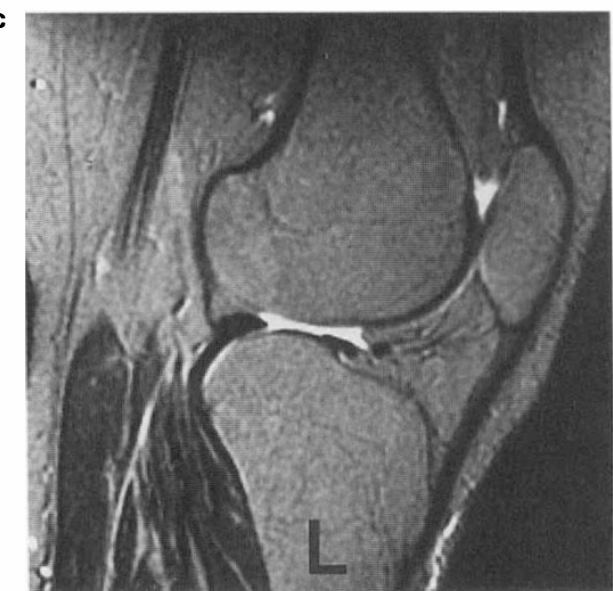

e

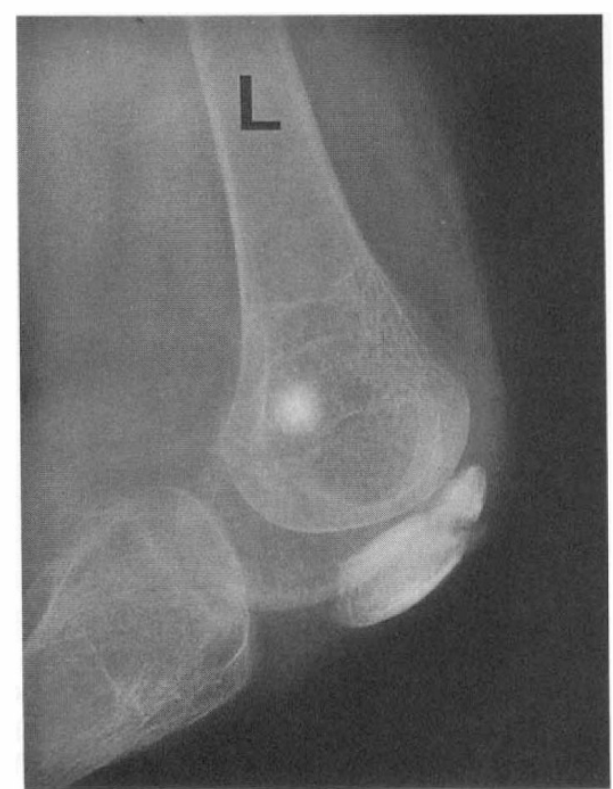

b

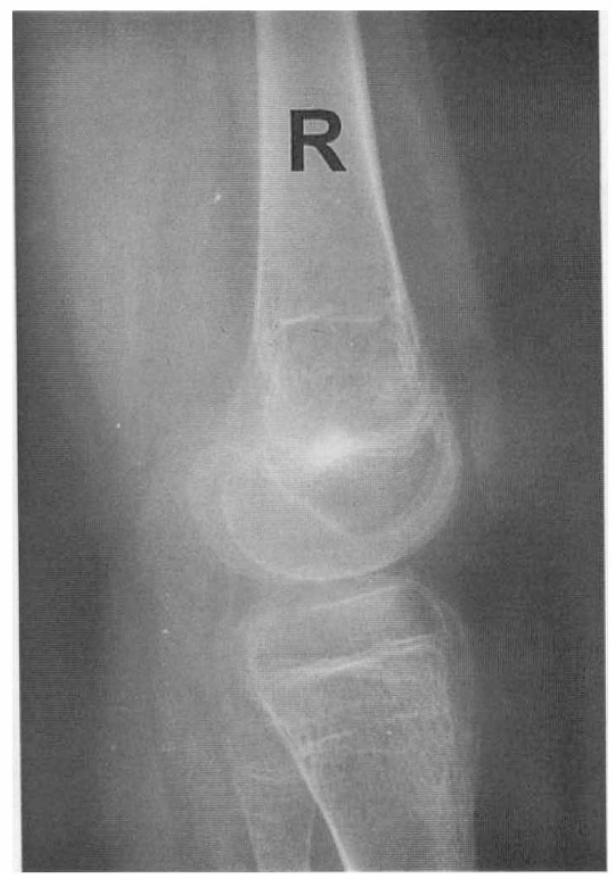

d

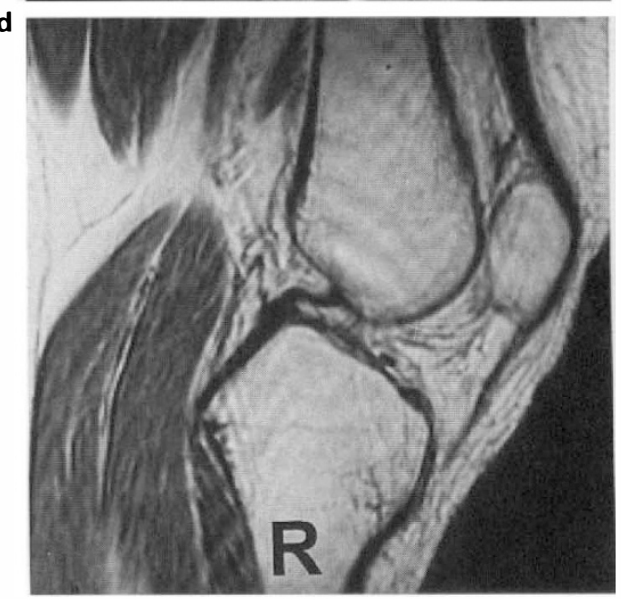

f

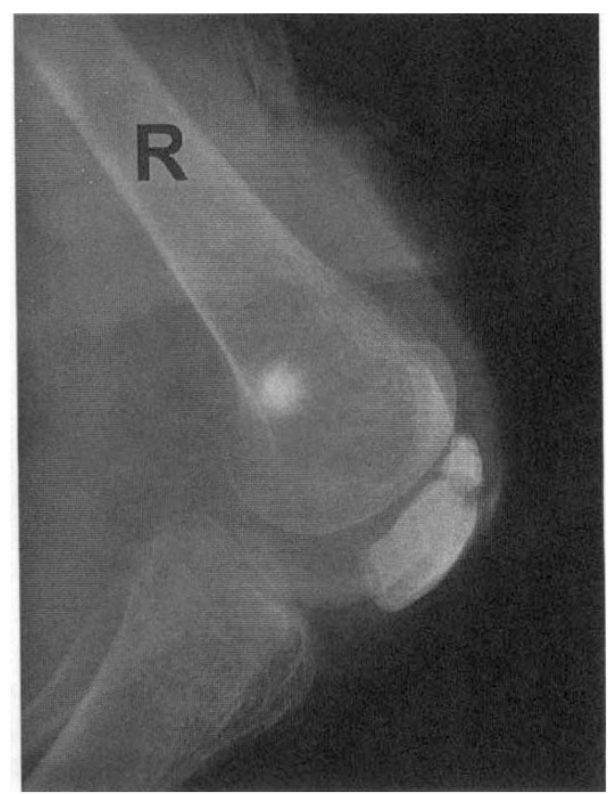



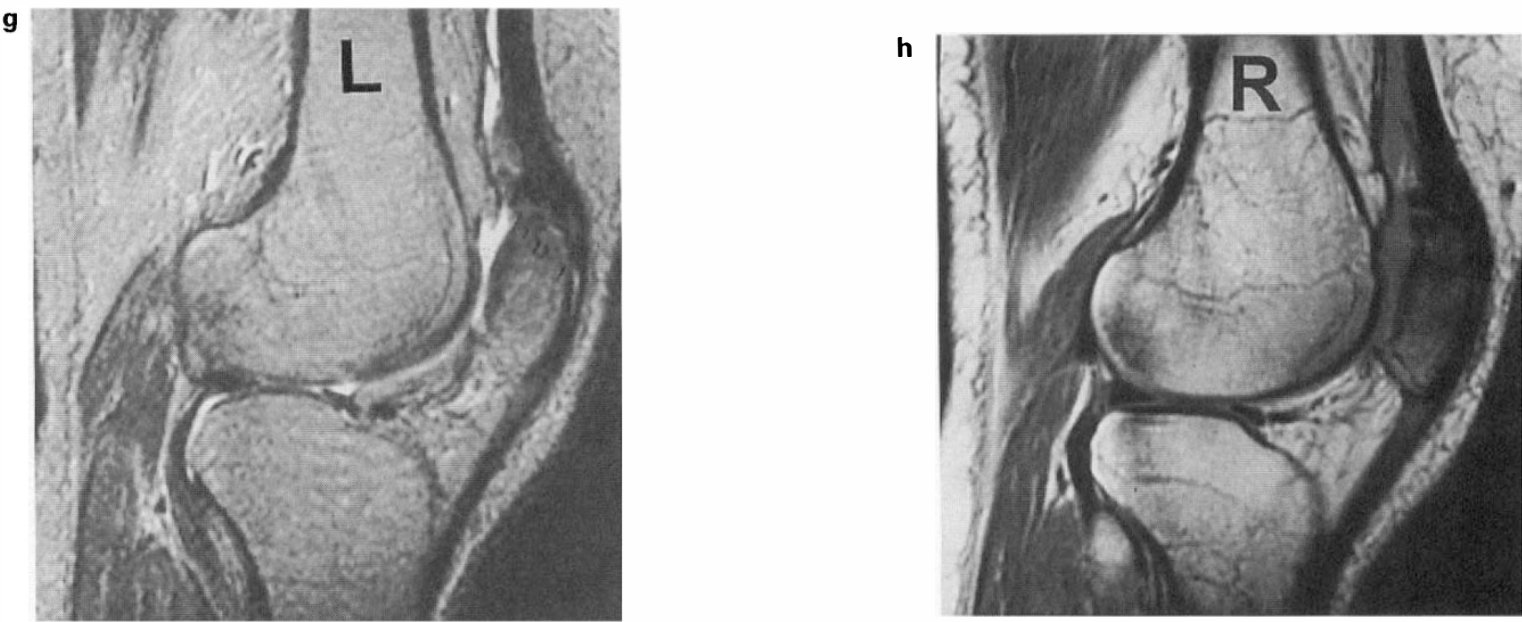

Figure 2 ( $\mathrm{a}$ and b) Pre-exercise lateral radiographs of both knees in an 11 year-old with a T1 level spinal cord injury. He started the FES program at 36 months post-injury at 13 years of age. Lateral radiographs taken at 13 years of age showed no fracture but were of poor quality and would not reproduce. (c and d) Pre-exercise sagittal MRIs (T2 weighted images, TR 2000) of each knee with no evidence of patellar fracture. (e and f) Several weeks after beginning the FES exercise program, the subject was noted to have bilateral knee effusions. These lateral radiographs of both knees revealed bilateral patellar tendon avulsion fractures of the proximal pole. (g and h) Sagittal MRIs (T2 weighted images, TR 2000) taken three days after the radiographs shown in $2 \mathrm{e} \& \mathrm{f}$ revealed evidence of bilateral patella tendon avulsion fractures

exercise. Case 10 had a consistently elevated value but never showed clinical, radiographic or MRI evidence of abnormalities. This patient has now been followed for 36 months without evidence of joint abnormalities (see Table 2).

Five subjects had synovial fluid samples of both knees and both ankles sent for CMGP testing by ELISA at the time of their arthroscopies. One subject (Case 14) had an abnormal value in her right knee of $23 \mu \mathrm{g} \mathrm{ml}^{-1}$ during her pre-exercise arthroscopy. This was associated with inflammatory reactive fibrosis seen on synovial biopsy from the same time. Her values were 0 on subsequent arthroscopic examinations.

There was no worsening of abnormal findings in pre-exercise studies, but significant improvement was seen in several instances, especially with effusions seen at MRI, arthroscopic chondromalacia in the ankle, and changes in the synovial joint lining.

\section{Subsequent adverse effects of FES on joints during exercise}

Three subjects developed joint swelling during the exercise program. One (Case 4), a former gymnast, developed clinical swelling of the left knee. The only abnormality noted in this subject was a small effusion, seen on the MRI scan of the left knee post-exercise, which was not present pre-exercise. The swelling was felt to be due to a minor capsular strain in a hyperextensible knee. The swelling resolved after cessation of the exercise program. The second subject (Case 17) developed a swollen ankle during the exercise program. Plain radiographs and tomograms were negative. A bone scan was performed to rule out a stress fracture and revealed generalized increased uptake in the right ankle joint region, but no fracture. Doppler studies were negative for deep venous thrombosis. A repeat MRI of the ankle revealed a partial tear of the deltoid ligament which was not present on the pre-exercise ankle MRI. This suggested that the swelling was due to an ankle sprain. After several weeks of rest, this subject was able to complete the exercise program and eventually progressed to the walking program without a recurrence of the swelling. Post-exercise and post standing and walking MRIs of the ankles were normal.

One subject (Case 19) developed bilateral clinical knee effusions a few weeks after beginning the exercise program. Follow-up radiographs and MRIs of both knees showed partial avulsion of the patellar tendons (Figures $2 \mathrm{a}-\mathrm{h}$ ). Following four weeks of rest, the swelling resolved and the patient was able to continue with the FES exercise program.

No new radiographic abnormalities were seen in the post-exercise studies. Five additional new effusions were revealed on post-exercise MRIs of the knee but none was clinically palpable. One subject (Case 12) had a small talar bone contusion on MRI of the right ankle in the post exercise study which was not seen on plain radiographs nor in the follow-up post standing and walking MRI studies. No new abnormalities were seen by knee or ankle arthroscopies or synovial biopsies.

Analysis of serum CMGP in the post-exercise studies revealed one subject (Case 18) with an elevated value of $23 \mu \mathrm{g} \mathrm{ml}^{-1}$ (normal $<20 \mu \mathrm{g} \mathrm{ml}^{-1}$ ). No clinical, radiographic, or MRI evidence of any 
a

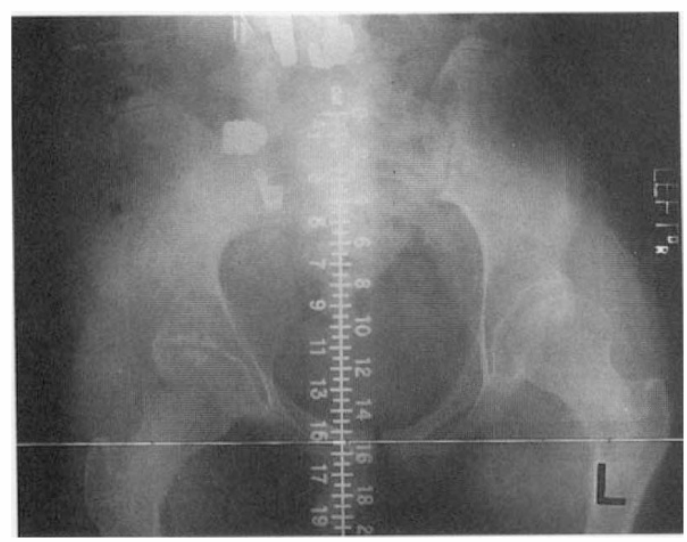

b

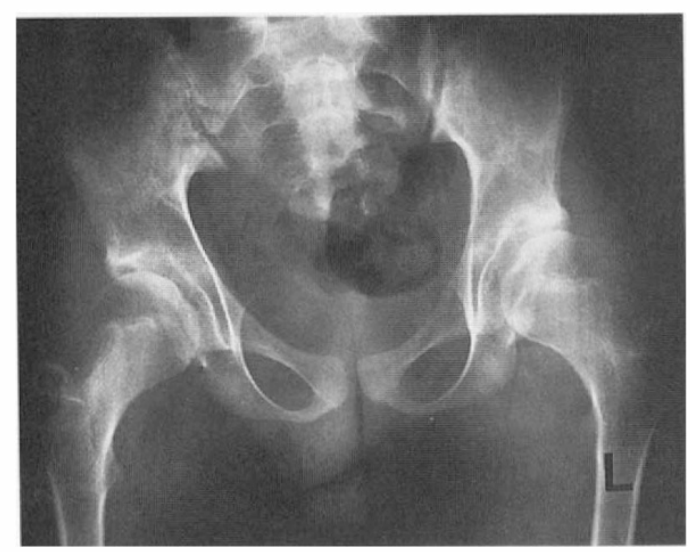

c

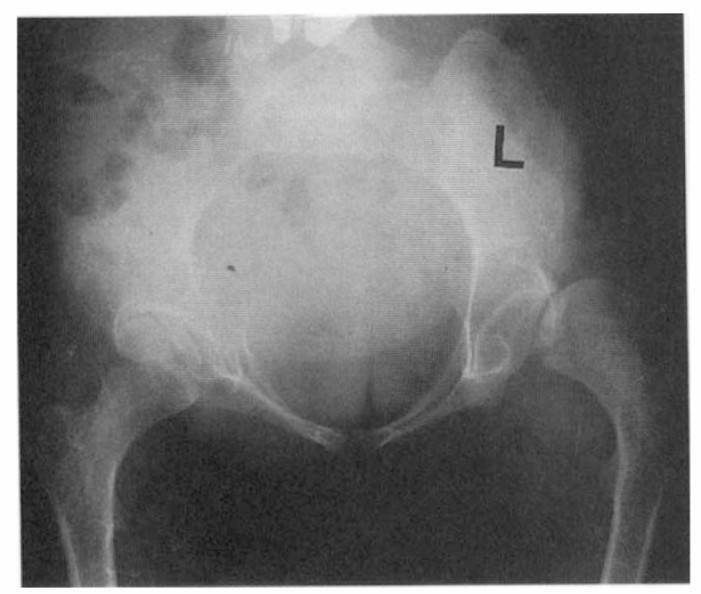

d
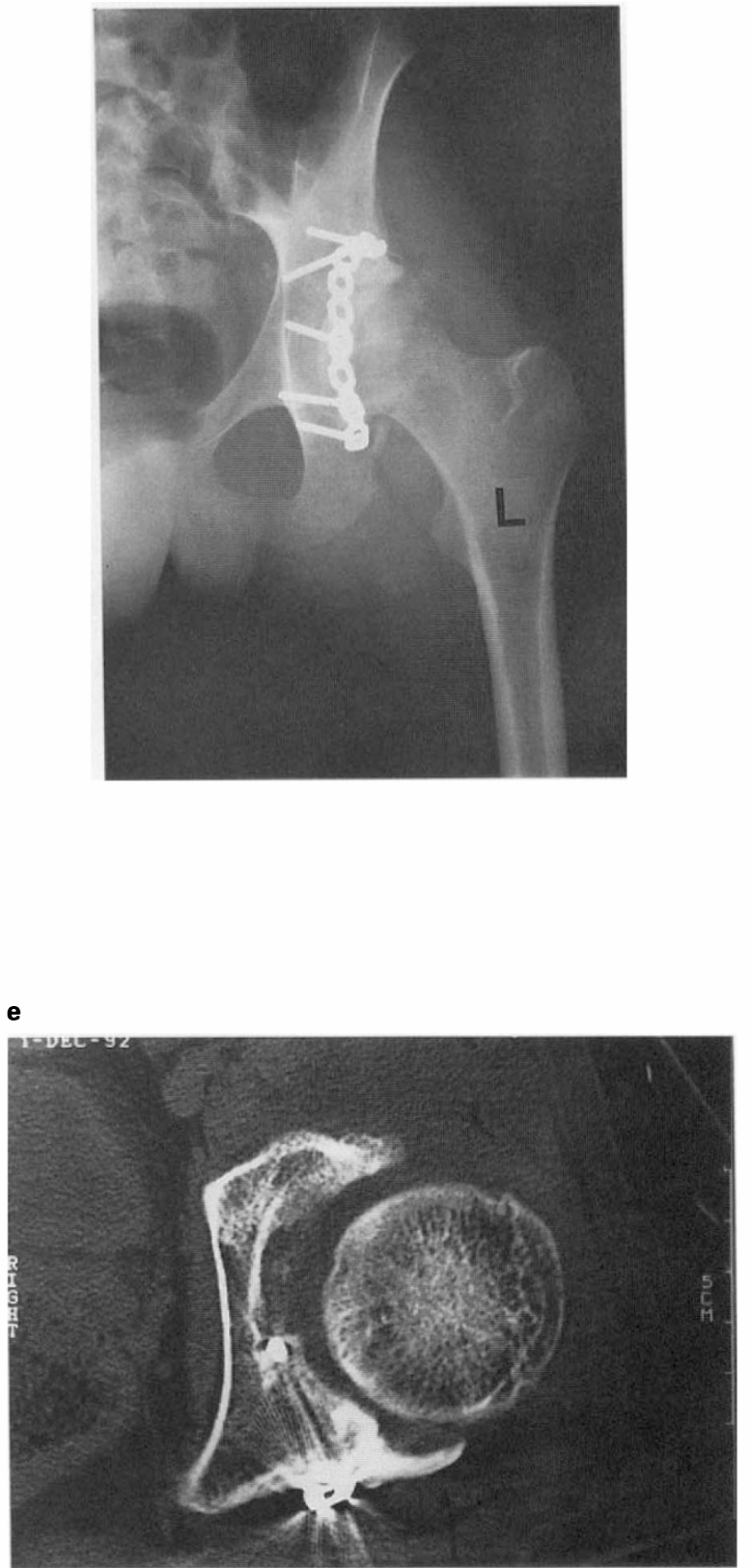

Figure 3 (a) This is the pre-exercise plain hip radiograph on Case 16, a 15-year-old boy with T8 paraplegia who was entered into the program six years post-injury. He is one year status post anterior and posterior spinal fusion with instrumentation for paralytic scoliosis with slight, residual pelvic obliquity between L4 and the sacrum. The left hip has a CE angle of 5 degrees, but Shenton's line was intact. Clinical examination of the hip revealed 20-degree hip flexion contractures and abduction of only 20 degrees bilaterally. (b) This radiograph was taken four months after Figure 3A, following the subject's participation in the exercise program. No significant changes were noted. The sclerotic bone density changes in the hip joints was felt to be due to different positioning with regards to pelvic rotation as noted by the difference in the obturator foramen. (c) Four weeks into the standing program, the subject was noted to have an increase in the pelvic obliquity upon standing, and the left hip was noted to be subluxed in a posterior-superior direction. (d) Following complete evaluation of the hip to rule out septic dislocation, the subject underwent a posterior reefing of the hip capsule and posterior iliac buttressing shelf with internal fixation. (e) A postoperative CT scan at nine months post-surgery demonstrated increased buttressing of the hip socket posteriorly. The space between the bone and the femoral head at the most lateral margin posteriorly has a fibrous capsule. It is anticipated that this will undergo fibrocartilaginous metaplasia 
abnormality could be found. Six months later the value was normal (Table 2). No abnormal values were seen in synovial fluid samples for CMGP post-exercise.

a
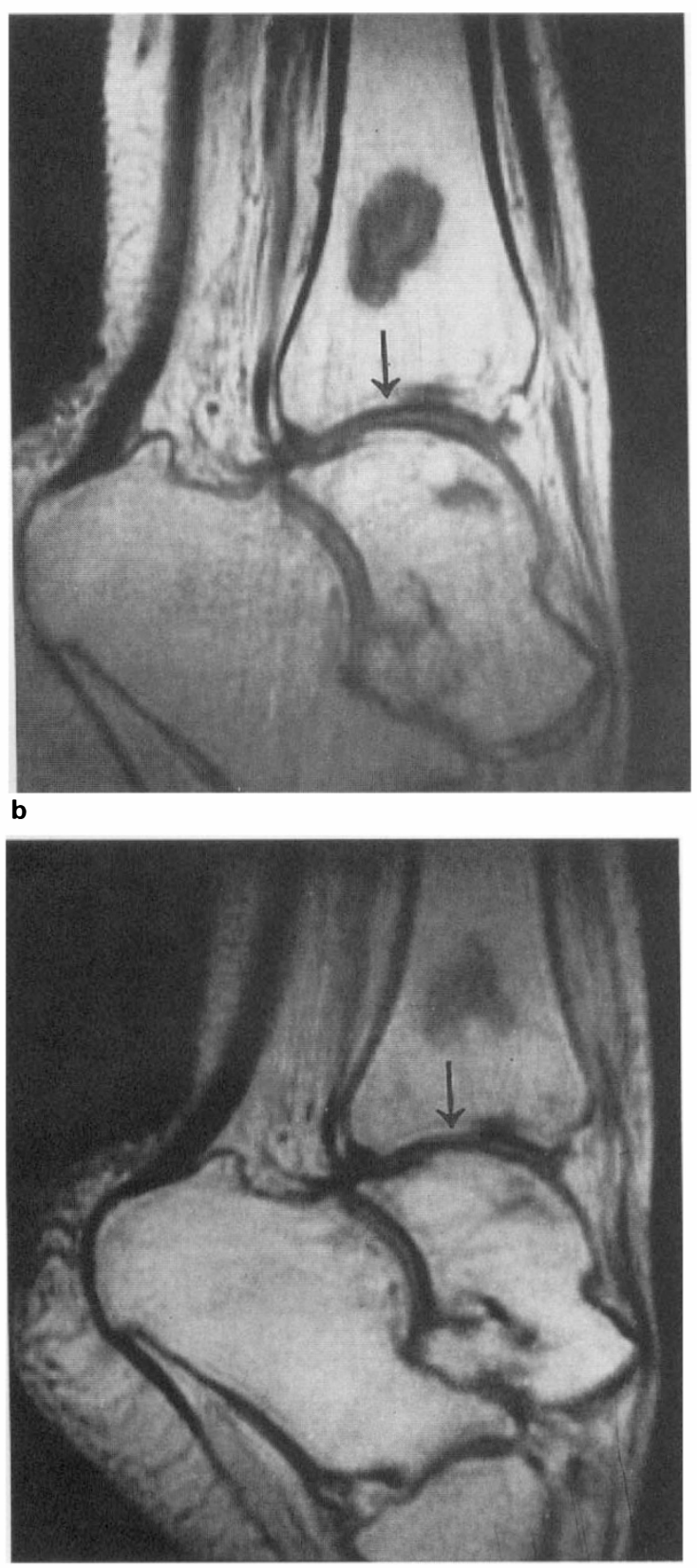

Figure 4 (a) Sagittal view of the left ankle showing a benign fibrous defect in the distal tibia (Case 10). Note the height of the articular cartilage. (b) This is an MRI six months following the MRI in Figure 4a. This was initially read as decreased joint space (arrow), but judging from the location of the fibrous defect and the shape of the talus, this is probably due to a different level image and not truly decreased joint space. The subject had no clinical effusion and no effusion by MRI, and this therefore was considered a false-positive reading
Subsequent adverse effects of FES following standing and walking

One subject (Case 16) with the pre-exercise hip at risk developed a left subluxed hip upon standing (Figures 3a through e). The pre- and post-exercise hip radiographs showed a hip at risk for subluxation with a $\mathrm{CE}$ angle of only 5 degrees (normal $>20$ degrees). An MRI revealed a large effusion of the hip joint which was aspirated and found to be sterile. The subject underwent a posterior shelf procedure to stabilize the hip. At 40 months post-surgery he has not shown a recurrence of hip subluxation and he is now standing in the FES program. No other new clinical or radiographic abnormalities were seen in the post standing and walking studies.

At the time of final post-standing and walking analysis in the nine subjects, 5 of 18 knees showed effusion on MRI. Compared to the pre-exercise studies of these 18 knees, the effusions were still present in three knees, had resolved in one knee, and new effusions were found in two knees. None of the effusions was clinically palpable, nor did they present any clinical problems. Despite a normal clinical knee examination, the post-standing and walking MRI of one subject (Case 18) revealed a strain of the anterior cruciate ligament which was not previously present.

Analysis of the ankle MRIs showed that one of 14 ankles had a minimal effusion post standing and walking which had been present preexercise. One subject (Case 10) had a decreased joint space in the tibiotalar joint on the post standing and walking MRI (Figures $4 \mathrm{a}$ and $\mathrm{b}$ ). Further review showed that this finding was probably due to different MRI parameters and variation in ankle position instead of true loss of joint space. The ankle MRI of one subject (Case 12) revealed a new distal tibia contusion post standing and walking. Radiographs were normal and radiographic follow-up at 2 years revealed no changes on plain radiographs.

Arthroscopic evaluation of the articular cartilage of the knees and ankles in five subjects revealed no abnormalities post standing and walking. Likewise, the synovial biopsies in these subjects post standing and walking revealed no additional abnormalities.

Despite absence of injury, an elevated serum CMGP level was revealed in one subject (Case 17) post standing and walking. This subject had a deltoid ligament sprain, but this occurred before her postexercise serum sample was taken. The etiology of the abnormal value after standing and walking is unclear (Table 2), and no long-term follow up on this subject is available.

\section{Discussion}

Prior to the initiation of the FES program in these subjects, the joints revealed few pre-existing abnormalities. Roentgenograms of the long bones showed advanced demineralization, most notable in the 
metaphyses. MRIs revealed minimal effusions located in the peripatellar region of 14 of the 30 knees. Two subjects had abnormalities on synovial biopsy consistent with Enneking's histological findings of fibrofatty connective tissue in the postmortem knee of a patient with a spinal cord injury. ${ }^{1}$ In all but one subject the MRIs revealed macroscopically normal appearing articular cartilage prior to the FES program. The paucity of abnormal findings which would be expected in this population may be due to the fact that individuals with a spinal cord injury still have motion in their joints despite their inability to bear weight. This joint motion occurs during daily range of motion exercises and transfers.

Although demineralization seen on radiographs did not change over the course of this study, a modest increase in bone mineral density as measured by dual photon absorptiometry has been realized in some of these same subjects following FES exercise. ${ }^{21}$

Pre-existing knee ligament injuries with resulting instability were a concern with one subject (Case 5). Despite severe instability, joint degeneration was not seen with FES. This affected only one subject, but it suggests that knee instability alone would not automatically contraindicate application of FES. Strengthening the quadriceps and the hamstrings are known to help to stabilize the knees ${ }^{22}$ after ligament injuries. Restrengthening a paralyzed lower extremity which has knee instability with FES-induced exercise may have a similar effect.

The most serious joint problem encountered in this study was a hip subluxation in one subject (Case 16). Prior to the onset of the study, this subject had 20degree hip flexion contractures bilaterally and marginal bony coverage of the femoral head $\left(\mathrm{CE}>20^{\circ}\right.$ is normal) which predisposed him to this complication.

The prevalence of hip instability in children with spinal cord injury is $40 \% .{ }^{23}$ Full hip range of motion and hip stability are now requirements for participation in the FES program. Those with fixed flexion or adduction contractures are not accepted into the program until the contractures are corrected by therapy or are surgically released. Although patients with myelodysplasia can ambulate with dislocated hips, they must do so in braces. However, with FES the need for braces is eliminated by stimulating the knee and hip extensors. We suspect that sufficient muscle power cannot be generated using FES without a stable proximal fulcrum (located hip).

Subjects in the FES program are carefully monitored for hip stability with standing radiographs of the hips prior to the program, upon initial standing, and every 6 to 12 months during the standing phase of FES. Hips with normal acetabular coverage did not develop subluxation with FES.

Case 10 (Figures $4 \mathrm{a}$ and $\mathrm{b}$ ) highlights a problem with MRI screening for articular joint damage. Since there is so much variability in exact scanning locations for comparing cartilage height, MRI may only be helpful in detecting large changes. None of the subjects in this study developed significant changes such as subchondral fractures or bone infarcts, which are easily documented on MRI.

New biochemical analysis may be a more accurate prognosticator of early joint changes. Numerous molecules have been examined as possible 'markers' for cartilage degeneration in humans and animals, including proteoglycans, glycosaminoglycans, collagen fragments, adhesion molecules, and other proteins. ${ }^{24}$ CMGP has been found in serum and synovial fluid from individuals with ongoing cartilage degradation, ${ }^{18-20}$ but its presence does not appear to be specific for osteoarthritis itself. ${ }^{25}$ The data accumulated to date indicate that no single entity will probably serve as a true marker specific for cartilage degeneration. It is possible, however, that a panel of such molecules would be helpful in future diagnostic evaluations ${ }^{26}$. The small number of subjects available for the CMGP assay makes the results of the study regarding concentration of this protein insufficient for statistical analysis. However, the trend suggests that release of CMGP from articular cartilage does not occur with FES, indicating that there is no significant degradation of the joint.

None of the subjects in the FES program developed a Charcot joint. One theory for the development of Charcot joints is that an insensate joint that is subjected to continuous activity can sustain articular fractures and cartilage breakdown, since the patient does not have sensation of pain associated with injury and continues to traumatize. ${ }^{27}$ In our study the subjects were followed for only 1 year; furthermore, the amount and duration of exercise, standing and walking in this FES program may be below the threshold required to precipitate changes. One subject (Case 10) has been followed with further radiographs and MRIs for 2 years without evidence of joint problems.

Another theory which has recently gained support is that an abnormality of the neurovascular mechanism may contribute to the development of Charcot arthropathy. ${ }^{28}$ This theory states that absence of sympathetic input which is often seen in peripheral neuropathy leads to increased bone-blood flow and active resorption of bone. This theory is supported by the lack of Charcot changes in this study of patients with insensate joints but apparently normal sympathetic nervous systems because these subjects have upper motor neuron lesions.

Although many subjects had small effusions detectable only by MRI, the presence of a clinical effusion in an individual with spinal cord injury may be the most important indicator of a joint problem. Three of the subjects in the exercise program developed clinical effusions. The cause of knee swelling in one subject was most likely due to minor strain in a hyperextensible knee. The other subject who developed an ankle effusion had a partial deltoid ligament tear on MRI. Both effusions resolved with conservative treatment. One subject developed bilat- 
eral clinical knee effusions shortly after beginning the exercise program. Follow-up MRIs of both knees showed partial avulsion of the patellar tendons not seen on radiographs (Figure 2). Following rest, the swelling resolved and the subject was able to continue with FES exercise. All of the clinically apparent effusions were detectable within 12 weeks of starting the FES. Subjects participating in an FES program who develop clinically palpable joint effusions should be evaluated thoroughly for an etiology before continuing with FES.

In conclusion, many of the pre-existing changes in subjects with spinal cord injuries improved following mobilization of their joints with an FES exercise program. Subjects in the FES exercise and walking program did not show any evidence of joint destruction. Although this is very encouraging, it must be remembered that the joints were studied for only 1 year. Current long-term evaluation of joints in subjects standing with FES at home is underway. Routine weekly clinical exam for ankle and knee joint effusions are recommended for subjects beginning an FES program with MRIs and radiographs taken only as needed. Radiographs of the hips should be taken before participation, upon standing, and every 6 to 12 months thereafter until further long-term data are available.

\section{Acknowledgements}

The authors gratefully acknowledge Charles Cole, MD, for his review and preliminary analysis of radiographs, MRIs, and arthroscopic studies; Cecelia Mullin, PTA, for her help with data collection; and Carolyn Hendrix for editorial assistance and manuscript preparation.

\section{References}

1 Enneking WF, Horowitz M. The intra-articular effect of immobilization on the human knee. J Bone and Joint Surg 1972; 54A: $973-965$.

2 Akeson WH, Amiel D, Abel MF, Garfin S, Woo S. Effects of immobilization on joints. Clin Orthop 1987; 219: $28-36$.

3 Palmoski M, Perricone E, Brandt KD. Development and reversal of a proteoglycan aggregation defect in normal canine knee cartilage after immobilization. Arthr and Rheum 1979; 22(5): $508-517$.

4 Salter RB, Simmonds DF, Malcolm BW, et al. The biological effect of continuous passive motion on the healing of fullthickness defects in articular cartilage. An experimental investigation in the rabbit. J Bone and Joint Surg 1980; 62A: $1232-1251$

5 Palmoski MJ, Colyer RA, Brandt KD. (1980) Joint motion in the absence of normal loading does not maintain normal articular cartilage. Arthr and Rheum 1980;23(3): 325-334.

6 Palmoski MJ, Brandt KD. Running inhibits the reversal of atrophic changes in the canine knee cartilage after removal of a leg cast. Arthr and Rheum 1981; 24(11): 1329-1337
7 Jarvelin J, Kiviranta 1, Saamanen AM, Tammi M, Helminen HJ. Partial restoration of immobilization-induced softening of canine articular cartilage after remobilization of the knee (stifle) joint. $J$ Orthop Res 1989; 7: 352--358.

8 Katz 1, Rabinowitz JG, Dziadiw R. Early changes in Charcot's joints. Am J Roentgenol 1961; 86: $965-974$.

9 Marsolais EB, Kobetic R. Functional electrical stimulation for walking in paraplegia. $J$ Bone and Joint Surg 1987; 69A: 728-733

10 Smith BT, Betz RR, Mulcahey MJ, Triolo RJ. Reliability of percutaneous intramuscular electrodes for upper extremity functional neuromuscular stimulation in adolescents with C5 tetraplegia. Arch Phys Med Rehabil 1994; 75: 939 945.

11 Triolo RJ, Kobetic R, Betz RR (in press) Standing and Walking with FNS: Technical and Clinical Challenges. In: Harris G, editor. Human Motion Analysis. New York (In press).

12 Thomasson JL, Sundaram M. The diabetic foot. Radiographic appearances. Orthopedics 1985; 8(5): 670-676.

13 Sabiston CP, Adams ME, Li DKB. MRI of osteoarthrosis: correlation with gross pathology using an experimental model. $J$ Orthop Res 1987; 5: $164-172$

14 Crues JV III, Ryu R. MRI of the knee. Surg Rounds Orthop., 1989.

15 Fife RS, Brandt KD. Identification of a high-molecular weight $(>400$ 000) protein in hyaline cartilage. Biochem Biophys Acta 1984; 802: $506-514$.

16 Fife RS Identification of link proteins and a 116000 -dalton matrix protein in canine meniscal cartilage. Arch Biochem Biophys 1985; 240: 682-688.

17 Fife RS, Palmoski MJ, Brandt KD. Metabolism of a cartilage matrix glycoprotein by normal and osteoarthritic canine articular cartilage. Arthritis Rheum 1986; 29: 1256 - 1262.

18 Fife RS, Brandt KD. Cartilage matrix glycoprotein is present in serum in experimental canine osteoarthritis. J Clin Invest 1989; 84: $1432-1439$

19 Fife RS. Identification of cartilage matrix glycoprotein in synovial fluid in human osteoarthritis. Arthritis Rheum 1988; 31: $553-556$.

20 Fife RS, Rachow JW, Ryan LM. Cartilage matrix glycoprotein in plasma and synovial fluids: retention in synovial fluids containing basic calcium phosphate crystals. Calcif Tis Int 1994; 55: $100-$ 102.

21 Betz R R, Triolo RJ, Hermida VM, Moynahan M, Gardner ER, Maurer A, Cook SD, Bennett JT. (1991) The effects of functional neuromuscular stimulation on the bone mineral content in the lower limbs of spinal cord injured children. American Spinal Injury Association annual meeting, Seattle.

22 Giove TP, Miller SJ 3d, Kent BE, Sanford TL, Garrick JG. Nonoperative treatment of the torn anterior cruciate ligament. $J$ Bone and Joint Surg 1983; 65A: 184-192.

23 Pierre-Jacques H, Betz RR, Henzes J (1995) Hip instability in spinal cord injury. Poster exhibit presented at the American Academy of Orthopaedic Surgeons annual meeting, Orlando.

24 Heinegard D, Saxne T. Connective tissue macromolecules as markers for tissue processes in joint diseases. Eur $J$ Rheumatol Inflamm 1991; 11: 91 - 99.

25 Fife RS, Brandt KD, Braunstein EM, Myers SL, Katz BP, Ehlich J, Shelbourne KD, Kalasinski LA. The presence of cartilage matrix glycoprotein in serum as determined by immunolocation analysis is not a sensitive indicator of 'early' osteoarthritis of the knee. J Lab Clin Med 1991; 117: 332-338.

26 Fife RS. Imaging, arthroscopy, and 'markers'. Current Opinion in Rheumatology 4: $560-565$.

27 Johnson JTH. Neuropathic fractures and joint injuries. $J$ Bone and Joint Surg 1967; 49A: $1-30$.

28 Browner AC, Allmar RM. Pathogenesis of the neuropathic joint: Neurotraumatic vs. neurovascular. Radiology 1981; 139: 349 354 . 